\title{
Prognostic significance of pretreatment elevated platelet count in patients with colorectal cancer: a meta-analysis
}

\author{
Yu Long ${ }^{1}$, Ting Wang ${ }^{1,2}$, Qian $\mathrm{Gao}^{3}$, Chengya Zhou ${ }^{1}$ \\ ${ }^{1}$ Department of Medical Oncology, Sichuan Cancer Hospital \& Institute, Sichuan Cancer Center, School of Medicine, University \\ of Electronic Science and Technology of China, Chengdu, China \\ ${ }^{2}$ Lung Cancer Center, West China Hospital, Sichuan University, Chengdu, Sichuan, China \\ ${ }^{3}$ Oncology Department, Du Jiang Yan Medical Center, Chengdu, Sichuan, China \\ Correspondence to: Ting Wang, email: tw19811026@163.com \\ Keywords: colorectal cancer, platelet, thrombocytosis, prognosis, meta-analysis \\ Received: October 05, $2016 \quad$ Accepted: October 24, 2016 \\ Published: November 09, 2016
}

\section{ABSTRACT}

Background: The prognostic effect of pretreatment elevated platelet count remains controversial in colorectal cancer patients. We conducted this meta-analysis to evaluate the prognostic impact of it in these patients.

Methods: PubMed, EMBASE and Cochrane Library were searched and studies on the prognostic significance of pretreatment elevated platelet count in colorectal patients were identified. We performed the meta-analysis, using overall survival and disease-free survival as outcomes and presenting data with hazard ratio and its $\mathbf{9 5 \%}$ confidence interval. Heterogeneity among studies and publication bias were also evaluated.

Results: Thirty studies were included in the meta-analysis. Compared with normal platelet count, pretreatment elevated platelet count was associated with poorer overall survival (Hazard ratio $=1.837,95 \%$ confidence interval, 1.497 to 2.255 , $p$ $=0.000$ ) and poorer disease-free survival (Hazard ratio $=1.635,95 \%$ confidence interval, 1.237 to $2.160, p=0.001$ ) in patients with colorectal cancer. In subgroup analyses, pretreatment elevated platelet count was also associated with poorer overall survival and disease-free survival in most subgroups.

Conclusion: Pretreatment elevated platelet count was an independent prognostic factor of overall survival and disease-free survival in colorectal cancer patients. Largescale prospective studies and a validation study are warranted.

\section{INTRODUCTION}

Colorectal cancer is the third most commonly diagnosed cancer in males and the second in females, with an estimated 1.4 million cases and 693,900 deaths occurring in 2012 [1]. Several markers like carcinoembryonic antigen, $\mathrm{C}$-reactive protein, albumin and tumor necrosis factors have been reported as prognostic indicators of outcomes in patients with colorectal cancer. However, it is still difficult to predict the outcome of patients before treatment.

There is a growing body of evidence showing that elevated platelet count or thrombocytosis is associated with outcomes of colorectal cancer [2-10]. Early in 1998, Monreal $\mathrm{M}$ et al. reported a significant association between pre-operative platelet count and survival in patients with colorectal cancer. The following studies confirmed this association $[2,3,5,6]$. However, some other studies failed to demonstrate the association between pretreatment elevated platelet count and outcomes of colorectal cancer [11-16]. Considering the controversial evidence, we conduct a meta-analysis to evaluate whether pretreatment elevated platelet count is a prognostic marker of colorectal cancer.

\section{RESULTS}

Study selection

Electronic search identified 2604 potentially relevant references. Additional 5 references were further identified by checking the reference list. 2469 duplicates 
or clearly irrelevant references were excluded through reading the abstracts. 140 references were read in full and 106 references were excluded for irrelevance or lack of data on comparisons or outcomes. Four references were excluded for repeated data. Finally, 30 references fulfilled the inclusion criteria and provided data for the metaanalysis [2-31] (Figure 1).

\section{Characteristics of included studies}

All thirty included articles were cohort studies published from 1998 to 2016. This meta-analysis included
9123 patients. The quality score of included studies ranged from 6 to 8 stars. Hazard ratios of overall survival were available in 28 included studies and hazard ratios of disease-free survival were available in 15 included studies. Characteristics of the included studies are listed in Table 1.

\section{Prognostic impact of pretreatment elevated platelet count on overall survival}

Twenty-eight studies contributed data to the analyses of overall survival [2-7, 9, 11-31]. Significant

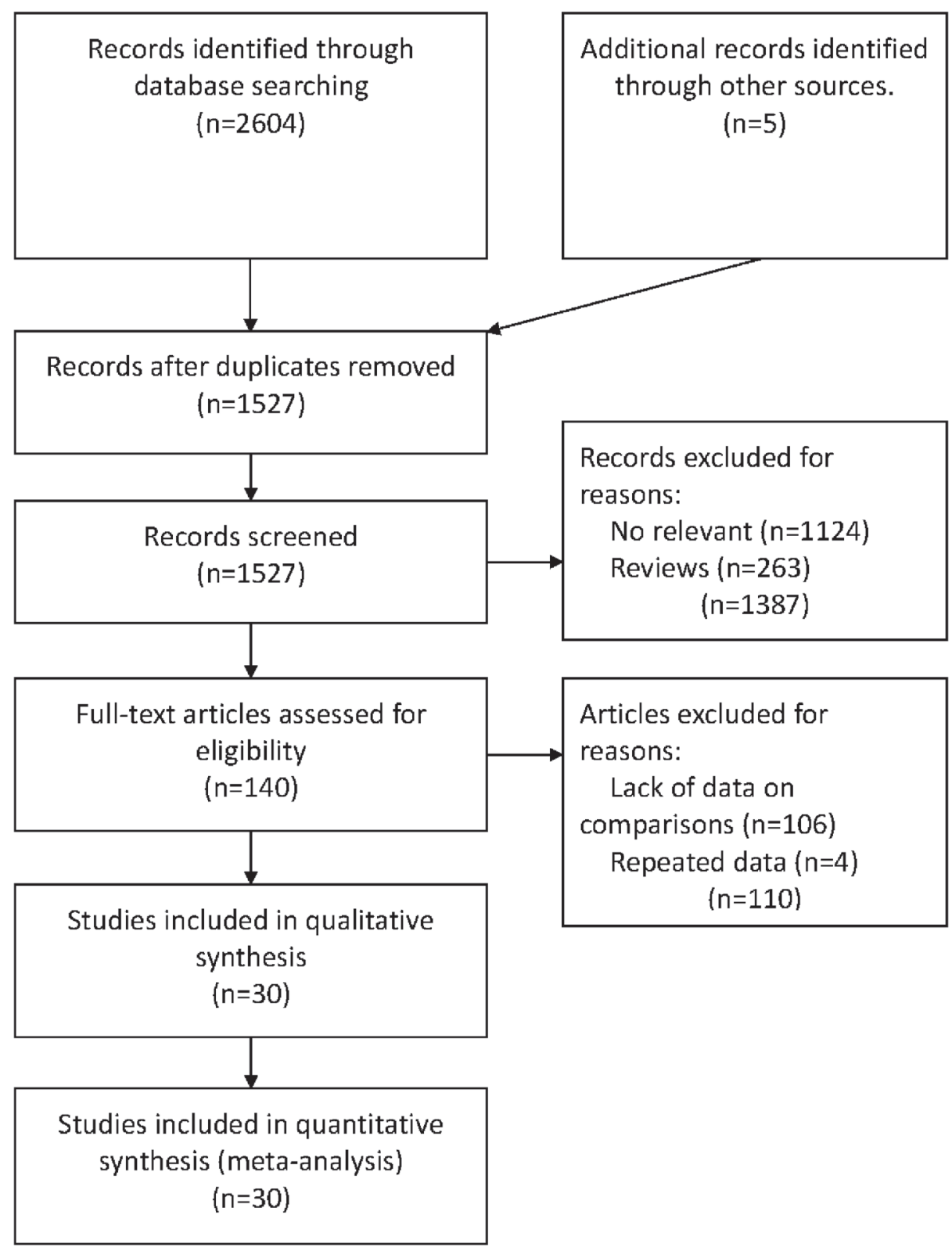

Figure 1: Flowchart of the process for the identification of relevant studies. 
Table 1: Characteristics of included studies

\begin{tabular}{|c|c|c|c|c|c|c|c|c|c|c|c|}
\hline Study & $\begin{array}{c}\text { Number } \\
\text { of } \\
\text { patients }\end{array}$ & $\begin{array}{l}\text { Tumor } \\
\text { type }\end{array}$ & $\begin{array}{c}\text { Disease } \\
\text { stage }\end{array}$ & $\begin{array}{l}\text { Cut-off } \\
\text { value }\end{array}$ & Area & $\begin{array}{l}\text { Study } \\
\text { time }\end{array}$ & $\begin{array}{c}\text { Age } \\
\text { (year) }\end{array}$ & $\begin{array}{c}\text { Follow-up } \\
\text { (year) }\end{array}$ & Treatment & Outcome & $\begin{array}{l}\text { Quality } \\
\text { score }\end{array}$ \\
\hline $\begin{array}{l}\text { Azab B } \\
2014[11]\end{array}$ & 580 & $\begin{array}{l}\text { colorectal } \\
\text { cancer }\end{array}$ & I-IV & tertile & USA & $\begin{array}{l}2005- \\
2011\end{array}$ & 68.6 & 40.5 & $\begin{array}{c}\text { surgery or } \\
\text { chemotherapy }\end{array}$ & $\begin{array}{c}\text { overall } \\
\text { survival, } \\
\text { disease- } \\
\text { free } \\
\text { survival }\end{array}$ & 6 \\
\hline $\begin{array}{l}\text { Baranyai Z } \\
2014 \text { [2] }\end{array}$ & 336 & $\begin{array}{l}\text { colorectal } \\
\text { cancer }\end{array}$ & I-IV & 400 & Hungary & $\begin{array}{l}2001- \\
2011\end{array}$ & 67 & 36.1 & surgery & $\begin{array}{l}\text { overall } \\
\text { survival, } \\
\text { disease- } \\
\text { free } \\
\text { survival }\end{array}$ & 8 \\
\hline $\begin{array}{l}\text { Carruthers } \\
\text { R } 2012 \\
{[17]}\end{array}$ & 115 & $\begin{array}{l}\text { rectal } \\
\text { cancer }\end{array}$ & I-III & NA & UK & $\begin{array}{l}2000- \\
2005\end{array}$ & 63.8 & 37.1 & $\begin{array}{l}\text { chemoradiation } \\
\text { and surgery }\end{array}$ & $\begin{array}{l}\text { overall } \\
\text { survival, } \\
\text { disease- } \\
\text { free } \\
\text { survival }\end{array}$ & 6 \\
\hline $\begin{array}{l}\text { Chen LL } \\
2016 \text { [3] }\end{array}$ & 503 & $\begin{array}{l}\text { colorectal } \\
\text { cancer }\end{array}$ & I-IV & 300 & China & $\begin{array}{l}2010- \\
2013\end{array}$ & 58 & 33.8 & surgery & $\begin{array}{l}\text { overall } \\
\text { survival }\end{array}$ & 7 \\
\hline $\begin{array}{l}\text { Choi KW } \\
2014 \text { [12] }\end{array}$ & 105 & $\begin{array}{l}\text { colorectal } \\
\text { cancer }\end{array}$ & I-IV & 400 & Korea & $\begin{array}{l}2005- \\
2008\end{array}$ & 63 & 44 & surgery & $\begin{array}{l}\text { overall } \\
\text { survival }\end{array}$ & 7 \\
\hline $\begin{array}{l}\text { Cravioto- } \\
\text { Villanueva } \\
\text { A } 2012 \text { [4] }\end{array}$ & 163 & $\begin{array}{l}\text { rectal } \\
\text { cancer }\end{array}$ & I-III & 350 & Mexico & $\begin{array}{l}2000- \\
2007\end{array}$ & 57.6 & 35.4 & $\begin{array}{c}\text { chemoradiation } \\
\text { and surgery }\end{array}$ & $\begin{array}{c}\text { overall } \\
\text { survival }\end{array}$ & 6 \\
\hline $\begin{array}{l}\text { Del Prete } \\
\text { M } 2015 \\
{[18]}\end{array}$ & 208 & $\begin{array}{l}\text { colorectal } \\
\text { cancer }\end{array}$ & IV & $\begin{array}{c}0.54 \\
\text { ULN }\end{array}$ & Italy & NA & 61 & NA & chemotherapy & $\begin{array}{c}\text { overall } \\
\text { survival }\end{array}$ & 6 \\
\hline $\begin{array}{l}\text { Guo T } 2014 \\
{[5]}\end{array}$ & 310 & $\begin{array}{l}\text { colorectal } \\
\text { cancer }\end{array}$ & I-III & 400 & USA & $\begin{array}{l}2004- \\
2013\end{array}$ & 69.9 & NA & NA & $\begin{array}{c}\text { overall } \\
\text { survival }\end{array}$ & 6 \\
\hline $\begin{array}{l}\text { Jósa V } \\
2015 \text { [19] }\end{array}$ & 336 & $\begin{array}{l}\text { colorectal } \\
\text { cancer }\end{array}$ & I-IV & 400 & Hungary & $\begin{array}{l}2001- \\
2011\end{array}$ & 66.9 & NA & $\begin{array}{c}\text { surgery and } \\
\text { chemotherapy }\end{array}$ & $\begin{array}{c}\text { overall } \\
\text { survival }\end{array}$ & 6 \\
\hline $\begin{array}{l}\text { Jósa V } \\
2015 \text { [19] }\end{array}$ & 166 & $\begin{array}{l}\text { colorectal } \\
\text { cancer }\end{array}$ & IV & 380 & Hungary & $\begin{array}{l}2001- \\
2011\end{array}$ & 62 & 28 & $\begin{array}{l}\text { surgery and } \\
\text { chemotherapy }\end{array}$ & $\begin{array}{c}\text { overall } \\
\text { survival, } \\
\text { disease- } \\
\text { free } \\
\text { survival }\end{array}$ & 6 \\
\hline $\begin{array}{l}\text { Kandemir } \\
\text { EG } 2005 \\
{[7]}\end{array}$ & 198 & $\begin{array}{l}\text { colon } \\
\text { cancer }\end{array}$ & I-II & 400 & Turkey & NA & 57 & 47 & NA & $\begin{array}{c}\text { overall } \\
\text { survival, } \\
\text { disease- } \\
\text { free } \\
\text { survival }\end{array}$ & 7 \\
\hline $\begin{array}{l}\text { Kaneko M } \\
2012 \text { [20] }\end{array}$ & 50 & $\begin{array}{l}\text { colorectal } \\
\text { cancer }\end{array}$ & IV & 400 & Japan & $\begin{array}{l}2005- \\
2010\end{array}$ & 61 & 17 & chemotherapy & $\begin{array}{c}\text { overall } \\
\text { survival, }\end{array}$ & 7 \\
\hline $\begin{array}{l}\text { Kawai K } \\
2013[8]\end{array}$ & 108 & $\begin{array}{l}\text { rectal } \\
\text { cancer }\end{array}$ & I-IV & 365 & Japan & $\begin{array}{l}2003- \\
2012\end{array}$ & 63.3 & 22.5 & $\begin{array}{l}\text { chemoradiation } \\
\text { and surgery }\end{array}$ & $\begin{array}{l}\text { disease- } \\
\text { free } \\
\text { survival }\end{array}$ & 7 \\
\hline $\begin{array}{l}\text { Kim HJ } \\
2015 \text { [9] }\end{array}$ & 314 & $\begin{array}{l}\text { rectal } \\
\text { cancer }\end{array}$ & I-III & 370 & Japan & $\begin{array}{l}2003- \\
2011\end{array}$ & NA & 36 & $\begin{array}{l}\text { chemoradiation } \\
\text { and surgery }\end{array}$ & $\begin{array}{c}\text { overall } \\
\text { survival, } \\
\text { disease- } \\
\text { free } \\
\text { survival }\end{array}$ & 7 \\
\hline
\end{tabular}

(Continued) 


\begin{tabular}{|c|c|c|c|c|c|c|c|c|c|c|c|}
\hline Study & $\begin{array}{c}\text { Number } \\
\text { of } \\
\text { patients }\end{array}$ & $\begin{array}{l}\text { Tumor } \\
\text { type }\end{array}$ & $\begin{array}{c}\text { Disease } \\
\text { stage }\end{array}$ & $\begin{array}{l}\text { Cut-off } \\
\text { value }\end{array}$ & Area & $\begin{array}{c}\text { Study } \\
\text { time }\end{array}$ & $\begin{array}{c}\text { Age } \\
\text { (year) }\end{array}$ & $\begin{array}{l}\text { Follow-up } \\
\text { (year) }\end{array}$ & Treatment & Outcome & $\begin{array}{l}\text { Quality } \\
\text { score }\end{array}$ \\
\hline $\begin{array}{l}\text { Kozak MM } \\
2015[21]\end{array}$ & 129 & $\begin{array}{l}\text { colorectal } \\
\text { cancer }\end{array}$ & I-III & 400 & USA & $\begin{array}{l}2005- \\
2009\end{array}$ & 67 & 24.7 & chemotherapy & $\begin{array}{c}\text { overall } \\
\text { survival, } \\
\text { disease- } \\
\text { free } \\
\text { survival }\end{array}$ & 7 \\
\hline $\begin{array}{l}\text { Kronborg } \\
\text { CS } 2015 \\
{[13]}\end{array}$ & 314 & $\begin{array}{l}\text { colorectal } \\
\text { cancer }\end{array}$ & IV & 400 & Denmark & $\begin{array}{l}2007- \\
2011\end{array}$ & 64.5 & 21.3 & chemotherapy & $\begin{array}{c}\text { overall } \\
\text { survival }\end{array}$ & 7 \\
\hline $\begin{array}{l}\text { Lee YS } \\
2016[22]\end{array}$ & 284 & $\begin{array}{l}\text { colorectal } \\
\text { cancer }\end{array}$ & II & 450 & Korea & $\begin{array}{l}2003- \\
2009\end{array}$ & 65 & 7.67 & $\begin{array}{l}\text { Adjuvant } \\
\text { therapy }\end{array}$ & $\begin{array}{l}\text { overall } \\
\text { survival, } \\
\text { disease- } \\
\text { free } \\
\text { survival }\end{array}$ & 8 \\
\hline $\begin{array}{l}\text { Lin MS } \\
2012[23]\end{array}$ & 150 & $\begin{array}{l}\text { colorectal } \\
\text { cancer }\end{array}$ & I-IV & 300 & China & $\begin{array}{l}2006- \\
2011\end{array}$ & 60.9 & NA & surgery or NA & $\begin{array}{c}\text { overall } \\
\text { survival }\end{array}$ & 6 \\
\hline $\begin{array}{l}\text { Monreal M } \\
1998[24]\end{array}$ & 180 & $\begin{array}{c}\text { colorectal } \\
\text { cancer }\end{array}$ & I-III & quertile & Spain & $\begin{array}{l}1994- \\
1996\end{array}$ & 67 & 13 & $\begin{array}{l}\text { surgery and } \\
\text { chemotherapy }\end{array}$ & $\begin{array}{c}\text { overall } \\
\text { survival }\end{array}$ & 7 \\
\hline $\begin{array}{l}\text { Neal CP } \\
2015[25]\end{array}$ & 302 & $\begin{array}{l}\text { colorectal } \\
\text { cancer }\end{array}$ & IV & 400 & UK & $\begin{array}{l}2006- \\
2010\end{array}$ & 64.8 & 29.7 & surgery & $\begin{array}{l}\text { overall } \\
\text { survival }\end{array}$ & 7 \\
\hline $\begin{array}{l}\text { Paik KY } \\
2014[26]\end{array}$ & 600 & $\begin{array}{c}\text { colorectal } \\
\text { cancer }\end{array}$ & I-IV & 400 & Korea & $\begin{array}{l}2006- \\
2009\end{array}$ & 62.3 & 27.4 & surgery & $\begin{array}{c}\text { overall } \\
\text { survival, } \\
\text { disease- } \\
\text { free } \\
\text { survival }\end{array}$ & 8 \\
\hline $\begin{array}{l}\text { Qiu MZ } \\
2010[27]\end{array}$ & 363 & $\begin{array}{l}\text { colorectal } \\
\text { cancer }\end{array}$ & I-IV & 400 & China & $\begin{array}{l}2005- \\
2009\end{array}$ & 56 & 26 & NA & $\begin{array}{l}\text { overall } \\
\text { survival }\end{array}$ & 7 \\
\hline $\begin{array}{l}\text { Roxburgh } \\
\text { CS } 2010 \\
{[28]}\end{array}$ & 287 & $\begin{array}{l}\text { colon } \\
\text { cancer }\end{array}$ & I-III & 400 & UK & $\begin{array}{l}1997- \\
2005\end{array}$ & NA & 65 & $\begin{array}{l}\text { surgery and } \\
\text { chemotherapy }\end{array}$ & $\begin{array}{c}\text { overall } \\
\text { survival }\end{array}$ & 7 \\
\hline $\begin{array}{l}\text { Sasaki K } \\
2012 \text { [29] }\end{array}$ & 636 & $\begin{array}{l}\text { colorectal } \\
\text { cancer }\end{array}$ & I-IV & 370 & Japan & $\begin{array}{l}2002- \\
2008\end{array}$ & 65.9 & 49.1 & $\begin{array}{l}\text { surgery and } \\
\text { chemotherapy }\end{array}$ & $\begin{array}{c}\text { overall } \\
\text { survival, } \\
\text { disease- } \\
\text { free } \\
\text { survival }\end{array}$ & 8 \\
\hline $\begin{array}{l}\text { Shen L } \\
2014[14]\end{array}$ & 199 & $\begin{array}{l}\text { rectal } \\
\text { cancer }\end{array}$ & II-III & 300 & China & $\begin{array}{l}2006- \\
2011\end{array}$ & 55 & 31 & $\begin{array}{c}\text { chemoradiation } \\
\text { and surgery }\end{array}$ & $\begin{array}{c}\text { overall } \\
\text { survival, } \\
\text { disease- } \\
\text { free } \\
\text { survival }\end{array}$ & 8 \\
\hline $\begin{array}{l}\text { Song A } \\
2015[15]\end{array}$ & 177 & $\begin{array}{l}\text { colorectal } \\
\text { cancer }\end{array}$ & IV & 400 & $\begin{array}{l}\text { South } \\
\text { Korea }\end{array}$ & $\begin{array}{l}2006- \\
2013\end{array}$ & 52 & 3.1 & chemotherapy & $\begin{array}{l}\text { overall } \\
\text { survival }\end{array}$ & 7 \\
\hline $\begin{array}{l}\text { Toiyama Y } \\
2015[30]\end{array}$ & 89 & $\begin{array}{l}\text { rectal } \\
\text { cancer }\end{array}$ & I-III & 300 & Japan & $\begin{array}{l}2001- \\
2012\end{array}$ & 65 & 56 & $\begin{array}{l}\text { chemoradiation } \\
\text { and surgery }\end{array}$ & $\begin{array}{c}\text { overall } \\
\text { survival, } \\
\text { disease- } \\
\text { free } \\
\text { survival }\end{array}$ & 8 \\
\hline
\end{tabular}

(Continued) 


\begin{tabular}{|c|c|c|c|c|c|c|c|c|c|c|c|}
\hline Study & $\begin{array}{c}\text { Number } \\
\text { of } \\
\text { patients }\end{array}$ & $\begin{array}{l}\text { Tumor } \\
\text { type }\end{array}$ & $\begin{array}{c}\text { Disease } \\
\text { stage }\end{array}$ & $\begin{array}{l}\text { Cut-off } \\
\text { value }\end{array}$ & Area & $\begin{array}{c}\text { Study } \\
\text { time }\end{array}$ & $\begin{array}{c}\text { Age } \\
\text { (year) }\end{array}$ & $\begin{array}{l}\text { Follow-up } \\
\text { (year) }\end{array}$ & Treatment & Outcome & $\begin{array}{c}\text { Quality } \\
\text { score }\end{array}$ \\
\hline $\begin{array}{l}\text { Wan S } \\
2013 \text { [31] }\end{array}$ & 1513 & $\begin{array}{l}\text { colorectal } \\
\text { cancer }\end{array}$ & I-IV & 400 & USA & $\begin{array}{l}1990- \\
2010\end{array}$ & 64.9 & 54 & $\begin{array}{c}\text { surgery and } \\
\text { chemotherapy }\end{array}$ & $\begin{array}{c}\text { overall } \\
\text { survival, } \\
\text { disease- } \\
\text { free } \\
\text { survival }\end{array}$ & 8 \\
\hline $\begin{array}{l}\text { Wei Y } 2015 \\
{[10]}\end{array}$ & 286 & $\begin{array}{l}\text { colorectal } \\
\text { cancer }\end{array}$ & II-III & 276 & China & $\begin{array}{l}2003- \\
2011\end{array}$ & 62 & 34 & $\begin{array}{c}\text { surgery and } \\
\text { chemotherapy }\end{array}$ & $\begin{array}{l}\text { disease- } \\
\text { free } \\
\text { survival }\end{array}$ & 7 \\
\hline $\begin{array}{l}\text { Zhao H } \\
2016[16]\end{array}$ & 122 & $\begin{array}{c}\text { colorectal } \\
\text { cancer }\end{array}$ & IV & 300 & China & $\begin{array}{l}2006- \\
2009\end{array}$ & NA & NA & chemotherapy & $\begin{array}{c}\text { overall } \\
\text { survival }\end{array}$ & 7 \\
\hline
\end{tabular}

NA, not available; ULN, upper limits of normal.

heterogeneity was found among studies $\left(\mathrm{I}^{2}=81 \%, \mathrm{p}=\right.$ 0.000 , Figure 2). Random-effect model was used. The pooled hazard ratio estimate showed that patients with pretreatment elevated platelet count had poorer overall survival compared with normal platelet count $(\mathrm{HR}=$ $1.837,95 \%$ confidence interval, 1.497 to $2.255, \mathrm{p}=0.000$, Figure 2).

We performed subgroup analyses on confounding factors such as disease stage, cancer type, cut-off values, etc. The results consistently showed that patients with pretreatment elevated platelet count had poorer overall survival compared with normal platelet count in most subgroups: multivariate analysis subgroup $(p=0.000)$, univariate analysis subgroup $(\mathrm{p}=0.000)$, preoperative subgroup $(\mathrm{p}=0.000)$, metastatic disease subgroup $(p=0.027)$, stage I-III disease subgroup $(p=0.000)$, rectal cancer subgroup $(p=0.009)$, cut-off value $\geq 400$ subgroup ( $p=0.000), 300 \leq$ cut-off value $<400$ subgroup $(p=0.000)$, cut-off value $<300$ subgroup $(p=0.043)$. In colon cancer subgroup, overall survival of patients with pretreatment elevated platelet count had no difference with patients with normal platelet count $(p=0.099)$. The detailed results of subgroup analyses were summarized in Table 2 .

\section{Prognostic impact of pretreatment elevated platelet count on disease-free survival}

Fifteen studies contributed data to the analyses of disease-free survival. Significant heterogeneity was found among studies $\left(I^{2}=82 \%, p=0.000\right.$, Figure 3$)$. Randomeffect model was used. The pooled hazard ratio estimate showed that patients with pretreatment elevated platelet count had poorer disease-free survival compared with normal platelet count $(\mathrm{HR}=1.635,95 \%$ confidence interval, 1.237 to $2.160, p=0.001$, Figure 3).

The results of subgroup analyses revealed that colorectal patients with pretreatment elevated platelet count had poorer disease-free survival compared with normal platelet count in the following subgroups: multivariate analysis subgroup $(p=0.007)$, preoperative subgroup $(\mathrm{p}=0.000)$, stage I-III disease subgroup $(\mathrm{p}=$ $0.007)$, rectal cancer subgroup $(\mathrm{p}=0.003)$, colon cancer subgroup ( $\mathrm{p}=0.001), 300 \leq$ cut-off value $<400$ subgroup $(\mathrm{p}=0.000)$, cut-off value $<300$ subgroup $(\mathrm{p}=0.003)$. In three subgroups, patients with pretreatment elevated platelet count had similar disease-free survival compared with patients with normal platelet count: univariate analysis subgroup $(p=0.056)$, cut-off value $\geq 400$ subgroup $(\mathrm{p}=0.056)$ and metastatic disease subgroup (all patients received $\mathrm{R} 0$ resection of primary and metastasis tumors $)(p=1.320)$. The detailed results of subgroup analyses were summarized in Table 2 .

\section{Publication bias}

Visual inspection of the funnel plot for overall survival and disease-free survival outcomes did not show the typically asymmetry associated with publication bias (Figure 4, Figure 5). Evidence of publication bias was also not seen with the Begg's tests of overall survival ( $p=$ $0.441)$ and disease-free survival $(\mathrm{p}=1.000)$.

\section{Sensitivity analyses}

The result of Sensitivity analyses demonstrated that no individual study had excessive influence on the stability of the pooled effect of comparisons for overall survival (Figure 6) and disease-free survival (Figure 7). The result of this meta-analysis is robust.

\section{DISCUSSION}

Elevated platelet count or thrombocytosis is observed in patients with various kinds of cancer and reported inversely correlated with survival [24, 32-36]. 
It was reported that nearly $10 \%$ to $30 \%$ of patients with colorectal cancer had elevated platelet count before treatment and worse survival than those with normal platelet count $[2-5,7,9,21]$. In our study, the results demonstrated that elevated platelet count was associated with shorter overall survival and disease-free survival in patients with colorectal cancer. Subgroup analyses demonstrated that the impact of elevated platelet count are consistent in different disease stages, tumor locations and analysis models. The result of our study is robust. This evidence indicated that platelet could be a simple and robust prognostic marker to identify high risk patients. Those patients should be taken into account to receive adjuvant therapy or maybe anti-platelet medications. There have been accumulating evidence that postdiagnosis aspirin therapy can improve overall survival of patients with colorectal cancer in recent years [37].

It is noteworthy that the cut-off values applied in included studies were not unified. Most studies used $400 \times$ $10^{9} / \mathrm{L}$ as cut-off value and some studies used $300 \times 10^{9} / \mathrm{L}$. The optimal cut-off value, however, was not validated in previous studies. The subgroup analyses reached consistent results among different cut-off value subgroup.
The cut-off value $\geq 400 \times 10^{9} / \mathrm{L}$ and between 300 and 400 can both distinguished patients well by overall survival and disease-free survival. Only in cut-off value $\geq 400 \times 10^{9} /$ L subgroup, platelet count failed to predict the disease-free survival of patients $(p=0.056$, Table 2$)$. Regarding this, it may be reasonable to suggest that the cut-off value between 300 and $400 \times 10^{9} / \mathrm{L}$ be applied in further investigation.

The mechanisms of tumor-related elevated platelet count or thrombocytosis remain undetermined. One of the potential hypotheses is that thrombocytosis is usually associated with inflammatory cytokines induced by interactions between tumor and host. Among these cytokines, IL-6, having multiple functions in many physiological conditions, plays a very important role in the formation of thrombocytosis [38]. By stimulating differentiation of megakaryocytes to platelets in the bone marrow, IL-6 induced thrombocytosis in various malignancies [39]. For another explanation of cancerassociated thrombocytosis, tumor can stimulate activation of platelet. As reported in several studies that cancer cells can secrete vascular endothelial growth factor to stimulates megakaryocyte differentiation [40]. For the prognostic association between elevated platelet count

\begin{tabular}{lcccccc} 
Overall survival & \multicolumn{7}{c}{ Hazard ratio and 95\% Cl } & Z-value & p-value & Weight \\
Study name & 0.850 & 0.544 & 1.329 & -0.713 & 0.476 & 4.745 \\
Azab B 2014 & 1.717 & 1.039 & 2.836 & 2.110 & 0.035 & 4.480 \\
Baranyai Z 2014 & 2.200 & 1.176 & 4.116 & 2.467 & 0.014 & 3.895 \\
Carruthers R 2012 & 1.814 & 1.056 & 3.116 & 2.158 & 0.031 & 4.294 \\
Chen LL 2016 & 1.266 & 0.434 & 3.691 & 0.432 & 0.666 & 2.292 \\
Choi KW 2014 & 5.650 & 1.551 & 20.582 & 2.625 & 0.009 & 1.779 \\
Cravioto-Villanueva A 2012 & 1.496 & 1.013 & 2.210 & 2.025 & 0.043 & 5.015 \\
Del Prete M 2015 & 1.800 & 0.991 & 3.270 & 1.930 & 0.054 & 4.030 \\
Guo T 2014 & 1.800 & 1.101 & 2.943 & 2.344 & 0.019 & 4.530 \\
Josa V 2015 & 2.840 & 0.969 & 8.323 & 1.903 & 0.057 & 2.278 \\
Jósa V 2015 & 5.361 & 1.555 & 18.485 & 2.659 & 0.008 & 1.891 \\
Kandemir EG 2005 & 5.020 & 1.690 & 14.911 & 2.905 & 0.004 & 2.242 \\
Kaneko M 2012 & 5.443 & 1.984 & 14.931 & 3.290 & 0.001 & 2.462 \\
Kim HJ 2015 & 0.999 & 0.994 & 1.004 & -0.392 & 0.695 & 6.132 \\
Kozak MM 2015 & 0.900 & 0.659 & 1.229 & -0.663 & 0.507 & 5.370 \\
Kronborg CS 2015 & 7.027 & 1.035 & 47.695 & 1.995 & 0.046 & 0.963 \\
Lee YS 2016 & 1.962 & 1.172 & 3.284 & 2.564 & 0.010 & 4.416 \\
Lin MS 2012 & 2.467 & 1.117 & 5.450 & 2.233 & 0.026 & 3.194 \\
Monreal M 1998 & 1.451 & 0.864 & 2.437 & 1.407 & 0.159 & 4.401 \\
Neal CP 2015 & 1.403 & 0.500 & 3.937 & 0.643 & 0.520 & 2.397 \\
Paik KY 2014 & 3.475 & 1.564 & 7.721 & 3.058 & 0.002 & 3.172 \\
Qiu MZ 2010 & 1.640 & 0.936 & 2.874 & 1.728 & 0.084 & 4.198 \\
Roxburgh CS 2010 & 3.040 & 1.841 & 5.019 & 4.347 & 0.000 & 4.483 \\
Sasaki K 2012 & 0.655 & 0.258 & 1.663 & -0.890 & 0.374 & 2.700 \\
Shen L 2014 & 1.618 & 0.960 & 2.727 & 1.808 & 0.071 & 4.385 \\
Song A 2015 & 4.960 & 1.829 & 13.452 & 3.146 & 0.002 & 2.496 \\
Toiyama Y 2015 & 1.540 & 1.191 & 1.991 & 3.292 & 0.001 & 5.591 \\
Wan S 2013 & 2.028 & 0.664 & 6.195 & 1.241 & 0.215 & 2.170 \\
Zhao H 2016 & 1.837 & 1.497 & 2.255 & 5.826 & 0.000 & \\
Overall effect (random) & 1.000 & & & & &
\end{tabular}

Figure 2: Forest plot showing the prognostic effect of pretreatment elevated platelet count on overall survival of colorectal cancer patients. ${ }^{*} \mathrm{CI}$ : Confidence interval. 
Table 2: Summarized results of meta-analysis

\begin{tabular}{|c|c|c|c|c|c|c|c|c|c|}
\hline \multirow{3}{*}{$\begin{array}{l}\text { Outcomes } \\
\text { Overall survival }\end{array}$} & \multirow{3}{*}{$\begin{array}{c}\text { Subgroups } \\
\text { total }\end{array}$} & \multirow{3}{*}{$\begin{array}{c}\text { Analysis } \\
\text { Model }\end{array}$} & \multirow{3}{*}{$\begin{array}{c}\text { Studies } \\
28\end{array}$} & \multicolumn{2}{|c|}{ Heterogeneity } & \multirow{3}{*}{$\begin{array}{c}\begin{array}{c}\text { Hazard } \\
\text { Ratio }\end{array} \\
1.837\end{array}$} & \multirow{2}{*}{\multicolumn{2}{|c|}{$\begin{array}{c}95 \% \\
\text { Confidence } \\
\text { Interval }\end{array}$}} & \multirow{3}{*}{$\begin{array}{r}\text { P-value } \\
0.000\end{array}$} \\
\hline & & & & \multirow{2}{*}{$\begin{array}{c}\text { P-value } \\
0.000\end{array}$} & \multirow{2}{*}{$\begin{array}{c}\begin{array}{c}\text { I-square } \\
\text { \% }\end{array} \\
81.006\end{array}$} & & & & \\
\hline & & & & & & & 1.497 & 2.255 & \\
\hline & multivariable & random & 11 & 0.000 & 68.792 & 2.122 & 1.508 & 2.985 & 0.000 \\
\hline & univariable & fixed & 8 & 0.162 & 33.341 & 1.675 & 1.331 & 2.108 & 0.000 \\
\hline & preoperative & random & 20 & 0.000 & 83.380 & 2.015 & 1.547 & 2.625 & 0.000 \\
\hline & $\begin{array}{l}\text { metastatic } \\
\text { disease }\end{array}$ & random & 6 & 0.021 & 62.204 & 1.503 & 1.047 & 2.157 & 0.027 \\
\hline & $\begin{array}{l}\text { stage I-III } \\
\text { disease }\end{array}$ & random & 11 & 0.000 & 82.555 & 2.330 & 1.461 & 3.715 & 0.000 \\
\hline & rectal cancer & random & 5 & 0.008 & 70.832 & 2.796 & 1.286 & 6.078 & 0.009 \\
\hline & colon cancer & random & 2 & 0.088 & 65.731 & 2.594 & 0.837 & 8.035 & 0.099 \\
\hline & $\begin{array}{c}\text { cut-off value } \geq \\
400 \times 10^{9} / \mathrm{L}\end{array}$ & random & 15 & 0.000 & 77.687 & 1.633 & 1.284 & 2.077 & 0.000 \\
\hline & $\begin{array}{c}300 \leq \text { cut-off } \\
\text { value }<400 \times \\
10^{9} / \mathrm{L}\end{array}$ & random & 9 & 0.038 & 51.062 & 2.467 & 1.685 & 3.612 & 0.000 \\
\hline & $\begin{array}{c}\text { cut-off value }< \\
300 \times 10^{9} / \mathrm{L}\end{array}$ & - & 1 & - & - & 1.496 & 1.013 & 2.210 & 0.043 \\
\hline \multirow{11}{*}{$\begin{array}{l}\text { Disease-free } \\
\text { survival }\end{array}$} & total & random & 15 & 0.000 & 82.195 & 1.635 & 1.237 & 2.160 & 0.001 \\
\hline & multivariable & random & 6 & 0.000 & 90.188 & 2.166 & 1.234 & 3.802 & 0.007 \\
\hline & univariable & random & 9 & 0.008 & 61.329 & 1.377 & 0.992 & 1.909 & 0.056 \\
\hline & preoperative & random & 14 & 0.000 & 83.175 & 1.751 & 1.287 & 2.384 & 0.000 \\
\hline & $\begin{array}{l}\text { metastatic } \\
\text { disease }\end{array}$ & - & 1 & - & - & 0.850 & 0.540 & 1.320 & 0.459 \\
\hline & $\begin{array}{l}\text { stage I-III } \\
\text { disease }\end{array}$ & random & 8 & 0.000 & 78.468 & 1.697 & 1.154 & 2.495 & 0.007 \\
\hline & rectal cancer & Fixed & 5 & 0.194 & 34.037 & 1.588 & 1.175 & 2.147 & 0.003 \\
\hline & colon cancer & - & 1 & - & - & 4.102 & 1.822 & 9.235 & 0.001 \\
\hline & $\begin{array}{c}\text { cut-off value } \geq \\
400 \times 10^{9} / \mathrm{L}\end{array}$ & random & 6 & 0.000 & 81.695 & 1.589 & 0.989 & 2.555 & 0.056 \\
\hline & $\begin{array}{c}300 \leq \text { cut-off } \\
\text { value }<400 \times \\
10^{9} / \mathrm{L}\end{array}$ & Fixed & 6 & 0.076 & 49.840 & 2.030 & 1.599 & 2.578 & 0.000 \\
\hline & $\begin{array}{c}\text { cut-off value }< \\
300 \times 10^{9} / \mathrm{L}\end{array}$ & - & 1 & - & - & 1.853 & 1.236 & 2.780 & 0.003 \\
\hline
\end{tabular}

and patients' outcomes, there is a most widely accepted hypothesis that activated platelets contribute to the tumor growth, angiogenesis and metastasis by releasing various cytokines with inflammatory, proliferative and angiogenic activity [41-43]. With regard to tumor metastasis, platelets can cover and protect circulating tumor cells from the host's immune system. With these underlying mechanisms, platelets may be a direct or indirect target for cancer therapy. 
There are some limitations of this study. First, our analysis is based on low-level evidence retrospective studies, in most of which some important confounders were not well adjusted, such as tumor stage, therapeutic strategy or ratio of colon and rectal cancer. The result of subgroup analysis, however, demonstrated that the negative prognostic significance of thrombocytosis on overall survival and disease-free survival was consistent between groups. Subgroup analysis according to therapeutic strategies was not performed because of insufficient data. Second, the sample size of some included studies were very small. The results of subgroup analyses still confirm the prognostic significance of thrombocytosis. Third, although the platelet count is easy to measure, its utility as a clinical prognostic marker could be affected by some other conditions, such as thrombosis, coronary disease, splenic disease, myeloproliferative disease, blood coagulation disorders, iron deficiency anemia and drugs. Actually, some heterogeneity was unexplainable.

In conclusion, our study demonstrated that the pretreatment elevated platelet count was an independent prognostic factor of overall survival and disease-free survival in colorectal cancer patients. It may make sense

\begin{tabular}{lcccccc} 
Disease-free survival & \multicolumn{7}{c}{ Hazard ratio and 95\% Cl } & Z-value & p-value & Weight \\
Study name & 0.800 & 0.539 & 1.188 & -1.107 & 0.268 & 8.341 \\
Azab B 2014 & 2.000 & 1.206 & 3.317 & 2.686 & 0.007 & 7.535 \\
Baranyai Z 2014 & 1.600 & 0.850 & 3.011 & 1.457 & 0.145 & 6.613 \\
Carruthers R 2012 & 2.700 & 1.137 & 6.410 & 2.252 & 0.024 & 5.103 \\
Jósa V 2015 & 4.102 & 1.822 & 9.235 & 3.409 & 0.001 & 5.419 \\
Kandemir EG 2005 & 2.538 & 0.995 & 6.474 & 1.949 & 0.051 & 4.703 \\
Kawai K 2013 & 1.727 & 1.033 & 2.887 & 2.085 & 0.037 & 7.476 \\
Kim HJ 2015 & 1.010 & 1.000 & 1.020 & 1.970 & 0.049 & 10.018 \\
Kozak MM 2015 & 2.945 & 1.127 & 7.696 & 2.204 & 0.028 & 4.576 \\
Lee YS 2016 & 0.594 & 0.262 & 1.346 & -1.248 & 0.212 & 5.380 \\
Paik KY 2014 & 2.540 & 1.771 & 3.643 & 5.066 & 0.000 & 8.581 \\
Sasaki K 2012 & 0.813 & 0.417 & 1.586 & -0.607 & 0.544 & 6.359 \\
Shen L 2014 & 2.550 & 1.070 & 6.079 & 2.112 & 0.035 & 5.079 \\
Toiyama Y 2015 & 1.540 & 0.911 & 2.603 & 1.612 & 0.107 & 7.394 \\
Wan S 2013 & 1.865 & 1.108 & 3.140 & 2.345 & 0.019 & 7.423 \\
Wei Y 2015 & 1.635 & 1.237 & 2.160 & 3.455 & 0.001 & \\
Overall effect (random) & & & & & &
\end{tabular}

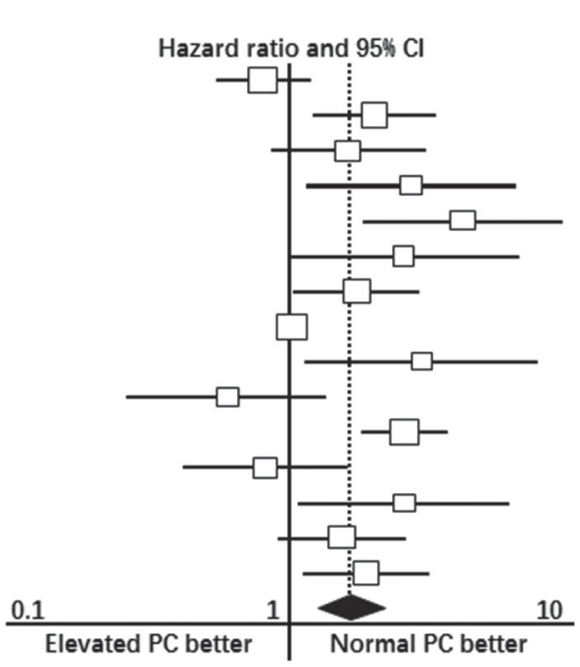

Figure 3: Forest plot showing the prognostic effect of pretreatment elevated platelet count on disease-free survival of colorectal cancer patients. ${ }^{*} \mathrm{CI}$ : Confidence interval.

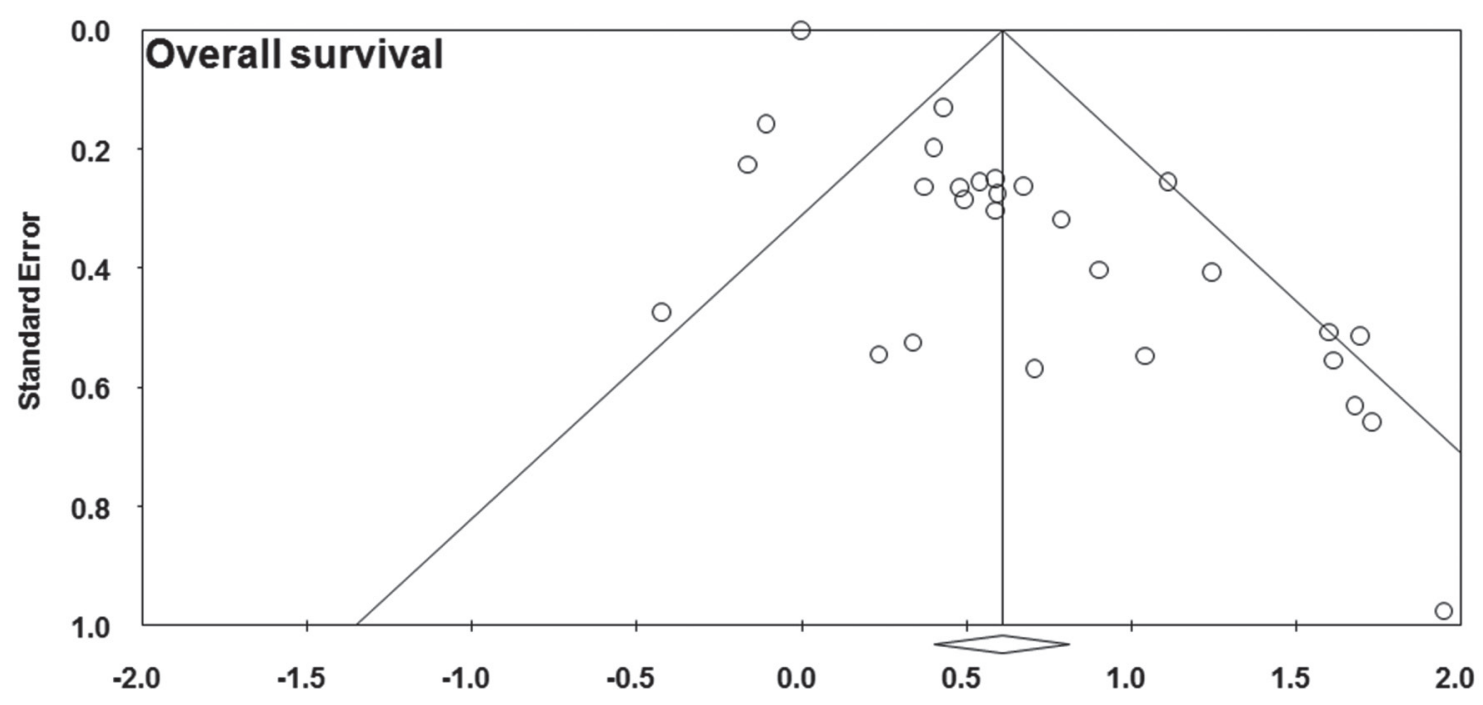

Figure 4: Funnel plot showing the publication bias of overall survival. 


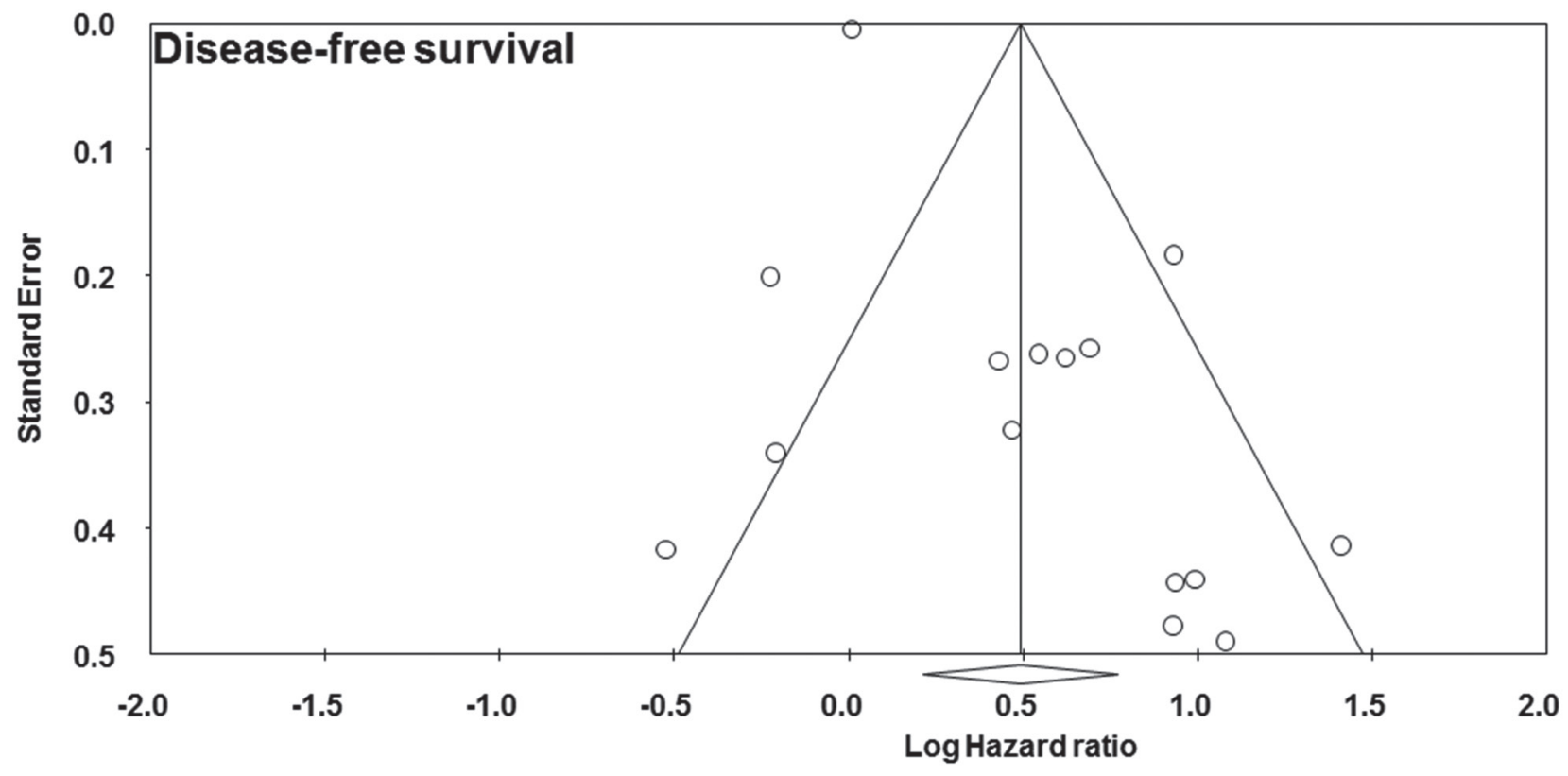

Figure 5: Funnel plot showing the publication bias of disease-free survival.

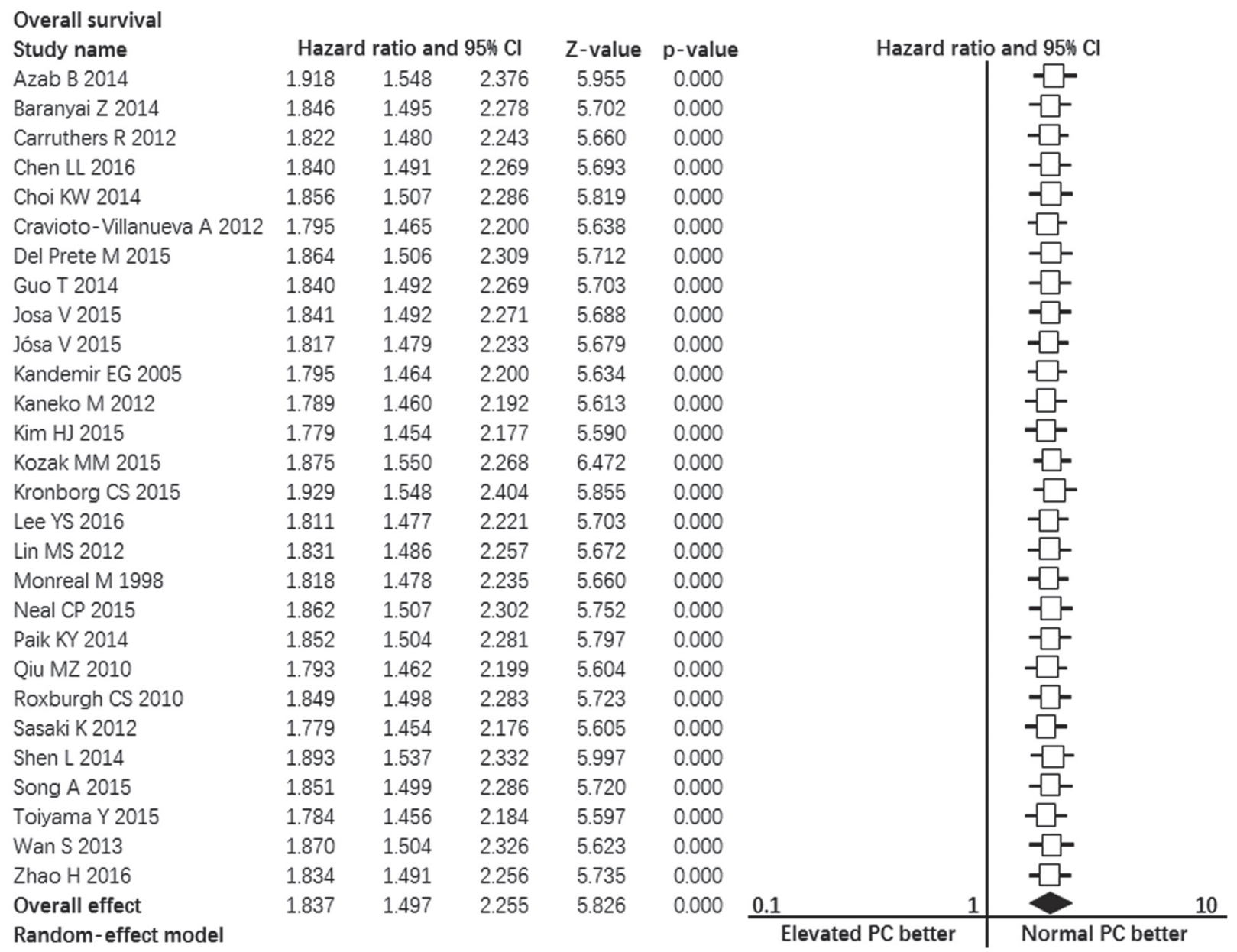

Figure 6: Forest plot showing the sensitivity analyses of overall survival. ${ }^{*} \mathrm{CI}$ : Confidence interval. 
that patients with elevated platelet count should receive intensive treatment or anti-platelet therapy. And large-scale prospective studies and a validation study are warranted to confirm its prognostic significance and determine the optimal platelet cut-off value.

\section{MATERIALS AND METHODS}

\section{Eligibility criteria}

This meta-analysis was performed according to the statement of Preferred Reporting Items for Systematic Reviews and Meta-Analyses (PRISMA) [44]. Cohort studies, being published from inception to July 9, 2016, which reported comparisons of overall survival or diseasefree survival between colorectal cancer patients with pretreatment elevated platelet count and those patients with pretreatment normal platelet count. The study participants had been pathologically diagnosed colorectal cancer patients.

\section{Search strategy}

An electronic search in PubMed, EMBASE and the Cochrane Library were conducted from inception to July 9, 2016. The following key words in combination as medical subject heading terms and text words were used: "colorectal cancer" and "platelet" or "thrombocytosis". Potentially relevant articles were identified by reading titles and abstracts. The full texts of the relevant articles were read to determine whether they met the inclusion criteria. The references were also searched to identify relevant studies.

\section{Quality assessment}

For cohort studies, the 9-star Newcastle-Ottawa Scale was used to assess the risk of bias [45]. This scale is an 8-item instrument that allows for assessment of patient population and selection, study comparability, follow-up, and outcome. Interpretation of the scale is performed by awarding points for high-quality elements. Studies with 5 or more stars were defined as high-quality studies and were included.

\section{Statistical analyses}

Data was extracted using a unified form and study information including author name, study year, study area, sample size, hazard ratio of overall survival or diseasefree survival were collected. If the hazard ratio was not reported in the original article, we would calculate hazard ratio from reported data according to the methods described by Tierney et al [46]. Statistical heterogeneity among studies was examined using the Cochrane $\mathrm{Q}$ test by calculating the $\mathrm{I}^{2}$ value [47]. The $\mathrm{I}^{2}$ value greater than $50 \%$ or $\mathrm{p}$ value less than 0.05 were considered to represent significant heterogeneity. The pooled hazard ratio and the $95 \%$ confidence interval were calculated using the $\mathrm{Z}$ test. The pooled hazard ratio and the $95 \%$ confidence interval were calculated using the Mantel-Haenszel formula (fixedeffect model) when heterogeneity was not detected ( $p>$ 0.05), or using the DerSimonian-Laird formula (randomeffect model) when heterogeneity was significant $(\mathrm{p}<$ 0.05 ) [48]. Publication bias was evaluated using the funnel plot and the Begg's test [49]. Influence analyses were conducted to access how robust the pooled estimators were by removing individual studies. An individual study

\begin{tabular}{|c|c|c|c|c|c|c|c|c|}
\hline \multirow{3}{*}{$\begin{array}{l}\text { Disease-free survival } \\
\text { Study name } \\
\text { Azab B } 2014\end{array}$} & \multirow{2}{*}{\multicolumn{3}{|c|}{ Hazard ratio and $95 \% \mathrm{Cl}$}} & \multirow{3}{*}{$\begin{array}{c}\text { Z-value } \\
3.565\end{array}$} & \multirow{3}{*}{$\begin{array}{c}\text { p-value } \\
0.000\end{array}$} & \multirow{2}{*}{\multicolumn{2}{|c|}{ Hazard ratio and $95 \% \mathrm{Cl}$}} & \\
\hline & & & & & & & & \\
\hline & 1.751 & 1.287 & 2.384 & & & & & \\
\hline Paik KY 2014 & 1.734 & 1.298 & 2.315 & 3.727 & 0.000 & & & \\
\hline Sasaki K 2012 & 1.550 & 1.187 & 2.025 & 3.216 & 0.001 & & & \\
\hline Shen L 2014 & 1.718 & 1.280 & 2.307 & 3.599 & 0.000 & & & \\
\hline Toiyama Y 2015 & 1.595 & 1.201 & 2.119 & 3.223 & 0.001 & & & \\
\hline Wan S 2013 & 1.646 & 1.224 & 2.215 & 3.293 & 0.001 & & & \\
\hline Wei Y 2015 & 1.619 & 1.209 & 2.168 & 3.232 & 0.001 & & & \\
\hline Baranyai Z 2014 & 1.608 & 1.203 & 2.149 & 3.210 & 0.001 & & & \\
\hline Carruthers R 2012 & 1.640 & 1.223 & 2.198 & 3.306 & 0.001 & & & \\
\hline Jósa V 2015 & 1.590 & 1.198 & 2.110 & 3.210 & 0.001 & & & \\
\hline Kandemir EG 2005 & 1.545 & 1.176 & 2.031 & 3.124 & 0.002 & & & \\
\hline Kawai K 2013 & 1.599 & 1.204 & 2.124 & 3.238 & 0.001 & & - & \\
\hline Kim HJ 2015 & 1.630 & 1.214 & 2.188 & 3.252 & 0.001 & & & \\
\hline Kozak MM 2015 & 1.721 & 1.297 & 2.283 & 3.765 & 0.000 & & & \\
\hline Lee YS 2016 & 1.588 & 1.198 & 2.105 & 3.213 & 0.001 & & & \\
\hline Overall effect & 1.635 & 1.237 & 2.160 & 3.455 & 0.001 & $\underline{0.1}$ & & 10 \\
\hline Random-effect model & & & & & & Elevated PC better & Norm & \\
\hline
\end{tabular}

Figure 7: Forest plot showing the sensitivity analyses of disease-free survival. *CI: Confidence interval. 
was suspected of excessive influence if the point estimate of its omitted analysis was outside the $95 \%$ confidence interval of the combined analysis. Statistical analyses were performed with Comprehensive Meta Analysis professional version 2.2 (Biostat Inc, Englewood NJ, www.meta-analysis.com).

\section{Abbreviations}

CI: Confidence interval; PRISMA: Preferred Reporting Items for Systematic Reviews and MetaAnalyses.

\section{ACKNOWLEDGMENTS AND FUNDING}

This research was supported by Provincial Research Project for Health Care of Cadres in Sichuan, China (Grant No. Chuan Gan Yan 2013-802).

\section{CONFLICTS OF INTEREST}

The authors declared no competing financial interest.

\section{Authors' contributions}

Conception/Design: Ting Wang, Yu Long. Data analysis and statistical guidance: Yu Long, Qian Gao, Chengya Zhou. Manuscript writing: Ting Wang, Yu Long. Final approval of the manuscript: Ting Wang, Yu Long, Chengya Zhou, Qian Gao.

\section{REFERENCES}

1. Torre LA, Bray F, Siegel RL, Ferlay J, Lortet-Tieulent J, Jemal A. Global cancer statistics, 2012. CA Cancer J Clin. 2015; 65:87-108. doi: 10.3322/caac.21262.

2. Baranyai Z, Krzystanek M, Josa V, Dede K, Agoston E, Szasz AM, Sinko D, Szarvas V, Salamon F, Eklund AC, Szallasi Z, Jakab F. The comparison of thrombocytosis and platelet-lymphocyte ratio as potential prognostic markers in colorectal cancer. Thromb Haemost. 2014; 111:483-90. doi: 10.1160/TH13-08-0632.

3. Chen LL, Zhang L, Li YL, Li XL, Liu WH, Yan J, Yang YF. [Association of preoperative platelet count with the prognosis of patients with colorectal cancer]. [Article in Chinese]. Nan Fang Yi Ke Da Xue Xue Bao. 2016; 36:482-7.

4. Cravioto-Villanueva A, Luna-Perez P, Gutierrez-de la Barrera M, Martinez-Gomez H, Maffuz A, Rojas-Garcia P, Perez-Alvarez C, Rodriguez-Ramirez S, RodriguezAntezana E, Ramirez-Ramirez L. Thrombocytosis as a predictor of distant recurrence in patients with rectal cancer. Arch Med Res. 2012; 43:305-11. doi: 10.1016/j. arcmed.2012.06.008.

5. Guo T, Krzystanek M, Szallasi Z, Szallasi A. Thrombocytosis portends adverse prognostic significance in patients with stage II colorectal carcinoma. F1000Res. 2014; 3:180. doi: 10.12688/f1000research.4856.2.

6. Josa V, Krzystanek M, Vass T, Lang T, Juhasz V, Szilagyi K, Tihanyi B, Harsanyi L, Szallasi Z, Salamon F, Baranyai Z. Thrombocytosis of Liver Metastasis from Colorectal Cancer as Predictive Factor. Pathol Oncol Res. 2015; 21:991-7. doi: 10.1007/s12253-015-9925-8.

7. Kandemir EG, Mayadagli A, Karagoz B, Bilgi O, Turken O, Yaylaci M. Prognostic significance of thrombocytosis in node-negative colon cancer. J Int Med Res. 2005; 33:228-35.

8. Kawai K, Kitayama J, Tsuno NH, Sunami E, Watanabe T. Thrombocytosis before pre-operative chemoradiotherapy predicts poor response and shorter local recurrence-free survival in rectal cancer. Int J Colorectal Dis. 2013; 28:52735. doi: 10.1007/s00384-012-1594-4.

9. Kim HJ, Choi GS, Park JS, Park S, Kawai K, Watanabe T. Clinical significance of thrombocytosis before preoperative chemoradiotherapy in rectal cancer: predicting pathologic tumor response and oncologic outcome. Ann Surg Oncol. 2015; 22:513-9. doi: 10.1245/s10434-014-3988-8.

10. Wei Y, Liang Z, Hong C, Luo D, Cai Z, Guan H, Zeng Z, Yang Z, Luo P, Tan Z. [Impact of platelet count on prognosis of stage II-III colorectal cancer receiving adjuvant chemotherapy]. [Article in Chinese]. Zhonghua Wei Chang Wai Ke Za Zhi. 2015; 18:1261-4.

11. Azab B, Mohammad F, Shah N, Vonfrolio S, Lu W, Kedia $\mathrm{S}$, Bloom SW. The value of the pretreatment neutrophil lymphocyte ratio vs. platelet lymphocyte ratio in predicting the long-term survival in colorectal cancer. Cancer Biomark. 2014; 14:303-12. doi: 10.3233/CBM-140416.

12. Choi KW, Hong SW, Chang YG, Lee WY, Lee B, Paik IW, Lee H. Inflammation-based score (Glasgow prognostic score) as an independent prognostic factor in colorectal cancer patients. Ann Surg Treat Res. 2014; 86:309-13. doi: 10.4174/astr.2014.86.6.309.

13. Kronborg CS, Jensen AR. Prognostic factors for overall survival in metastatic colorectal cancer using a stop-and-go FLIRI-based treatment strategy. Int J Colorectal Dis. 2015; 30:1059-65. doi: 10.1007/s00384-015-2264-0.

14. Shen L, Zhang H, Liang L, Li G, Fan M, Wu Y, Zhu J, Zhang Z. Baseline neutrophil-lymphocyte ratio $(>/=2.8)$ as a prognostic factor for patients with locally advanced rectal cancer undergoing neoadjuvant chemoradiation. Radiat Oncol. 2014; 9:295. doi: 10.1186/s13014-014-0295-2.

15. Song A, Eo W, Lee S. Comparison of selected inflammationbased prognostic markers in relapsed or refractory metastatic colorectal cancer patients. World J Gastroenterol. 2015; 21:12410-20. doi: 10.3748/wjg.v21.i43.12410. 
16. Zhao H, Wang Y, Yu J, Wei F, Cao S, Zhang X, Dong N, Li H, Ren X. Autologous Cytokine-Induced Killer Cells Improves Overall Survival of Metastatic Colorectal Cancer Patients: Results From a Phase II Clinical Trial. Clin Colorectal Cancer. 2016. doi: 10.1016/j.clcc.2016.02.005.

17. Carruthers R, Tho LM, Brown J, Kakumanu S, McCartney E, McDonald AC. Systemic inflammatory response is a predictor of outcome in patients undergoing preoperative chemoradiation for locally advanced rectal cancer. Colorectal Dis. 2012; 14:e701-7. doi: 10.1111/j.1463-1318.2012.03147.x.

18. Del Prete M, Giampieri R, Loupakis F, Prochilo T, Salvatore L, Faloppi L, Bianconi M, Bittoni A, Aprile G, Zaniboni A, Falcone A, Scartozzi M, Cascinu S. Prognostic clinical factors in pretreated colorectal cancer patients receiving regorafenib: implications for clinical management. Oncotarget. 2015; 6:33982-92. doi: 10.18632/ oncotarget.5053.

19. Josa V, Krzystanek M, Eklund AC, Salamon F, Zarand A, Szallasi Z, Baranyai Z. Relationship of postoperative thrombocytosis and survival of patients with colorectal cancer. Int J Surg. 2015; 18:1-6. doi: 10.1016/j. ijsu.2015.03.005.

20. Kaneko M, Nozawa H, Sasaki K, Hongo K, Hiyoshi M, Tada N, Murono K, Nirei T, Kawai K, Sunami E, Tsuno NH, Kitayama J. Elevated neutrophil to lymphocyte ratio predicts poor prognosis in advanced colorectal cancer patients receiving oxaliplatin-based chemotherapy. Oncology. 2012; 82:261-8. doi: 10.1159/000337228.

21. Kozak MM, von Eyben R, Pai JS, Anderson EM, Welton ML, Shelton AA, Kin C, Koong AC, Chang DT. The Prognostic Significance of Pretreatment Hematologic Parameters in Patients Undergoing Resection for Colorectal Cancer. Am J Clin Oncol. 2015. doi: 10.1097/ COC.0000000000000183.

22. Lee YS, Suh KW, Oh SY. Preoperative thrombocytosis predicts prognosis in stage II colorectal cancer patients. Ann Surg Treat Res. 2016; 90:322-7. doi: 10.4174/ astr.2016.90.6.322.

23. Lin MS, Huang JX, Zhu J, Shen HZ. Elevation of platelet count in patients with colorectal cancer predicts tendency to metastases and poor prognosis. Hepatogastroenterology. 2012; 59:1687-90. doi: 10.5754/hge12277.

24. Monreal M, Fernandez-Llamazares J, Pinol M, Julian JF, Broggi M, Escola D, Abad A. Platelet count and survival in patients with colorectal cancer--a preliminary study. Thromb Haemost. 1998; 79:916-8.

25. Neal CP, Cairns V, Jones MJ, Masood MM, Nana GR, Mann CD, Garcea G, Dennison AR. Prognostic performance of inflammation-based prognostic indices in patients with resectable colorectal liver metastases. Med Oncol. 2015; 32:144. doi: 10.1007/s12032-015-0590-2.

26. Paik KY, Lee IK, Lee YS, Sung NY, Kwon TS. Clinical implications of systemic inflammatory response markers as independent prognostic factors in colorectal cancer patients. Cancer Res Treat. 2014; 46:65-73. doi: 10.4143/ crt.2014.46.1.65.

27. Qiu MZ, Yuan ZY, Luo HY, Ruan DY, Wang ZQ, Wang $\mathrm{FH}$, Li YH, Xu RH. Impact of pretreatment hematologic profile on survival of colorectal cancer patients. Tumour Biol. 2010; 31:255-60. doi: 10.1007/s13277-010-0024-x.

28. Roxburgh CS, Wallace AM, Guthrie GK, Horgan PG, McMillan DC. Comparison of the prognostic value of tumour- and patient-related factors in patients undergoing potentially curative surgery for colon cancer. Colorectal Dis. 2010; 12:987-94. doi: 10.1111/j.1463-1318.2009.01961.x.

29. Sasaki K, Kawai K, Tsuno NH, Sunami E, Kitayama J. Impact of preoperative thrombocytosis on the survival of patients with primary colorectal cancer. World J Surg. 2012; 36:192-200. doi: 10.1007/s00268-011-1329-7.

30. Toiyama Y, Inoue Y, Kawamura M, Kawamoto A, Okugawa Y, Hiro J, Saigusa S, Tanaka K, Mohri Y, Kusunoki M. Elevated platelet count as predictor of recurrence in rectal cancer patients undergoing preoperative chemoradiotherapy followed by surgery. Int Surg. 2015; 100:199-207. doi: 10.9738/INTSURG-D-13-00178.1.

31. Wan S, Lai Y, Myers RE, Li B, Hyslop T, London J, Chatterjee D, Palazzo JP, Burkart AL, Zhang K, Xing J, Yang H. Preoperative platelet count associates with survival and distant metastasis in surgically resected colorectal cancer patients. J Gastrointest Cancer. 2013; 44:293-304. doi: 10.1007/s12029-013-9491-9.

32. Zeimet AG, Marth C, Muller-Holzner E, Daxenbichler G, Dapunt O. Significance of thrombocytosis in patients with epithelial ovarian cancer. Am J Obstet Gynecol. 1994; 170:549-54.

33. Ikeda M, Furukawa H, Imamura H, Shimizu J, Ishida H, Masutani S, Tatsuta M, Satomi T. Poor prognosis associated with thrombocytosis in patients with gastric cancer. Ann Surg Oncol. 2002; 9:287-91.

34. Gao J, Zhang HY, Xia YF. Increased platelet count is an indicator of metastasis in patients with nasopharyngeal carcinoma. Tumour Biol. 2013; 34:39-45. doi: 10.1007/ s13277-012-0508-y.

35. Silvis SE, Turkbas N, Doscherholmen A. Thrombocytosis in patients with lung cancer. JAMA. 1970; 211:1852-3.

36. Symbas NP, Townsend MF, El-Galley R, Keane TE, Graham SD, Petros JA. Poor prognosis associated with thrombocytosis in patients with renal cell carcinoma. BJU Int. 2000; 86:203-7.

37. Li P, Wu H, Zhang H, Shi Y, Xu J, Ye Y, Xia D, Yang J, Cai $\mathrm{J}$, Wu Y. Aspirin use after diagnosis but not prediagnosis improves established colorectal cancer survival: a meta-analysis. Gut. 2015; 64:1419-25. doi: 10.1136/ gutjnl-2014-308260.

38. Sierko E, Wojtukiewicz MZ. Platelets and angiogenesis in malignancy. Semin Thromb Hemost. 2004; 30:95-108. doi: 10.1055/s-2004-822974. 
39. Imai T, Koike K, Kubo T, Kikuchi T, Amano Y, Takagi M, Okumura N, Nakahata T. Interleukin-6 supports human megakaryocytic proliferation and differentiation in vitro. Blood. 1991; 78:1969-74.

40. Troxler M, Dickinson K, Homer-Vanniasinkam S. Platelet function and antiplatelet therapy. Br J Surg. 2007; 94:67482. doi: 10.1002/bjs.5852.

41. Dineen SP, Roland CL, Toombs JE, Kelher M, Silliman CC, Brekken RA, Barnett CC, Jr. The acellular fraction of stored platelets promotes tumor cell invasion. J Surg Res. 2009; 153:132-7. doi: 10.1016/j.jss.2008.04.013.

42. Kut C, Mac Gabhann F, Popel AS. Where is VEGF in the body? A meta-analysis of VEGF distribution in cancer. Br J Cancer. 2007; 97:978-85. doi: 10.1038/sj.bjc.6603923.

43. Sledge GW, Jr. Vascular endothelial growth factor in breast cancer: biologic and therapeutic aspects. Semin Oncol. 2002; 29:104-10.

44. Moher D, Liberati A, Tetzlaff J, Altman DG, Group P. Preferred reporting items for systematic reviews and meta-analyses: the PRISMA statement. PLoS Med. 2009; 6:e1000097. doi: 10.1371/journal.pmed.1000097.

45. Stang A. Critical evaluation of the Newcastle-Ottawa scale for the assessment of the quality of nonrandomized studies in meta-analyses. Eur J Epidemiol. 2010; 25:603-5. doi: 10.1007/s10654-010-9491-z.

46. Tierney JF, Stewart LA, Ghersi D, Burdett S, Sydes MR. Practical methods for incorporating summary time-toevent data into meta-analysis. Trials. 2007; 8:16. doi: 10.1186/1745-6215-8-16.

47. Higgins JP, Thompson SG. Quantifying heterogeneity in a meta-analysis. Stat Med. 2002; 21:1539-58. doi: 10.1002/ sim. 1186.

48. DerSimonian R, Laird N. Meta-analysis in clinical trials. Control Clin Trials. 1986; 7:177-88.

49. Begg CB, Mazumdar M. Operating characteristics of a rank correlation test for publication bias. Biometrics. 1994; 50:1088-101. 